\title{
A Plea for Critical Changes to the Electronic Medical Record
}

\author{
M. Ted Braid
}

The electronic medical record (EMR) is significantly more than a digitalized format for converting patient data into easily stored and recalled information. Today, each patient's EMR is a vast, growing accumulation of health data sourced from physicians and professional medical devices. And, according to HIPAA, it is the absolute property of the patient. At its best, the EMR is a trusted data source that contributes to improved patient care.

It is my concern that in its current state and considering existing legislation, the very thing we are building will fall in upon itself long before its potential is realized. It must change or the promise of national access to critical care data in the emergency department (ED), for example, may never be realized.

What is needed is a fundamental change in primary ownership (who holds the master record), how the electronic medical system is functionally assembled and used, and how this staggering amount of data is updated and verified to be current and accurate.

In support of this contention, we begin by demonstrating what we already face and where our policies and/or actions contribute more to ineffectiveness than improvement.

\section{Lack of Standards}

ICD10 (the 10th revision of the International Statistical Classification of Diseases and Related Health Problems) is a medical classification list by the World Health Organization. 1 It evolves to comply with newly discovered data. It replaced the ICD9 codes with no issues on our EMR, so standardization works. However with the data recorded in different EMR systems, the data fields are not standardized to data field name or even format of the data it contains. Information might be stored with a different name and in a different format from EMR to EMR. In practice, a patient with multiple specialists at different locations has multiple records, possibly stored differently enough from system to system that a third-party interface is needed to collect and analyze the whole data set. This risks error in data translation. As data grow, under the current lack of standards, 
critical data fall deeper into this validity crisis more rapidly than technology can correct using more complex validation methods.

To grasp this, one must understand the sheer mass of data and the predicted growth short term. Harmony Healthcare IT pegs the volume of healthcare data at 153 exabytes in 2013. A figure that will swell to 2,314 exabytes by 2020 .

At these projected growth rates, healthcare data are soon expected to reach the zettabyte and yottabyte scale. For reference sake, 1 zettabyte is equivalent to 152 million years of UHD (ultra-high definition) $8 \mathrm{~K}$ video format and 1.4 yottabytes, which is the largest decimal unit prefix in the metric system, is roughly equivalent to the mass of all the oceans. Further, about $80 \%$ of the world's healthcare data is unstructured, which means there is a lot of room for technology advances. ${ }^{2}$

Unstructured data are caused by lack of standardization. To put it in Harmony's frame of reference, the equivalent of $80 \%$ of the world's oceans would still carry on an oceanographic map the phrase, "There be Dragons." (Ancient ocean maps often had this or similar text over the area beyond which no ship had ventured.) "There be EMR dragons" where $80 \%$ of existing medical data resides. For all our work and expense, we still can only analyze $20 \%$ of these data. Add to this the cost for hours to learn and use the EMR, and there is the loss of an average of one patient visit day per location to overhead in order to enter and maintain data of which over three-quarters are unstructured.

Standards that structure data uniformly reduce the opportunity for error. Soliant Health states, the EMR has reduced the error rate in patient data by $3 \%$ to $4 \%$. This still leaves 195,000 patient-deaths due to these errors-more than AIDS, breast cancer, and auto accidents combined. ${ }^{3}$ While there is no data published to contend this $4 \%$ reductionwhich is a reduction of 7,800 souls - two factors are not considered in this statistic: errors in lab results often having the wrong result linked to the wrong patient and miscommunication among medical professionals both personally and recorded into the EMR. These errors add an additional 2,000 deaths a year! ${ }^{4}$ 


\section{Size is Not the Problem, and the Claims of Data Sharing are Misleading}

Over $75 \%$ of facilities share data to internal and external EMR systems. ${ }^{5}$ But this figure is limited to lab, radiology, and imaging data. Data supplied initially by agencies lead the way in providing universal secure access. Laboratory, pathology, and imaging companies have put in more technology, recommended and enacted better methods to share data, and designed solutions that are just now gaining attention.

\section{The CCDA}

CCDA (Consolidated Clinical Document Architecture) is discussed as a separate aspect to data sharing. It is the best format available but also faces the problems already discussed here, combined with a misplaced security and transport structure. An overview of five issues elucidates the problem.

\section{Complexity}

First, the 1-to-1 certificate required for security and encryption gives the physician the right to refuse to transmit data to a specific doctor when such a decision is the patient's or the courts. Using it is based on a $\mathrm{N}^{2}$ connection algorithm, where you must square the number of individual connections to securely send EHR data. If " $A$ " wants to connect to "B" there must be two connections for each location. And these must pass through the HIE (Health Information Exchange) or an HISP (Health Information Service Provider) again squaring the connection numbers. In other words, 47 centers in a county, having to select different HISP and HIE for any given reason could require up to 16,000,000 connections just to eventually share CCDA data between all of them. It is also a complicated, disparately configured nightmare that very often slows the import to a crawl trying to assimilate the transmitted data.

Second, the token and certificate a patient gets for their patient portal is far less complex and as secure. It must be, because unlike the CCDA, the portal gives access to complete demographics. Access to these data are analogous to handing someone your keys, wallet, and last tax return. In addition, the portal allows access to a patient's chart and the ability for patients to send a CCDA of their chart to any physician listed as approved to accept it. These defeat all the above, except that the version of the chart a patient sees is limited. 
Third, the CCDA is limited to transferring discrete data (text and numbers). The size of the CCDA remains very small compared to any other method. But again, there is no standard and some EMRs transfer these data as an Adobe Acrobat file. These files are infinitely more versatile but more difficult to extract discrete data, and they are severely limited as a file itself-simply an image in the database. Finally, it is huge compared to the XML (extensible mark-up language) standard CDDA for the same amount of data. CCDA's cannot transfer lab reports, imaging data, or any other type of object. These must be included as attachments, swelling the transmission from as small as $6 \mathrm{~Kb}$ to $260 \mathrm{Mb}$.

Fourth, CDDA content is directed by the physician releasing the data. It is their decision as to what to include, which may be half the data needed by the requesting physician.

The fifth and final massive complexity in sharing data is location. These exabytes of information are scattered among over 5000 hospitals and roughly 200,000 private practices, ambulatory surgical centers, and general practice physicians. Even considering that logically over $65 \%$ of these data are spread between hospitals with similar systems, it still faces the following challenges. The specific data that might be needed could reside anywhere in roughly 205,000 locations, and there is no standard to denote the location. Most critically the lack of standards, or cooperation to standardize among vendors prevents easy detection of duplicate records on the same patient between the two EMRs; increasing the risk of exchanging incorrect data. There is no way to verify and correct inaccurate/conflicting data transmitted. So, a mistake can grow quickly throughout systems, causing erroneous data declared good simply by its existing in more records.

This also leaves us with more potential good data, spread across over 200,000 locations that must be requested from each of these locations, not even knowing if the data exists before it can be transmitted. There is no way to verify validity, format, or fit of the data until after it is received. And considering the spread of data held individually and the limits of CCDA's transmission format, collection of thorough amounts of data for a single patient are not certain. Consider collecting massive specific data across a wide demographic to utilize this new gold mine of possibilities held as data by using any permutation of a 
system based on this existing model. This is the source of my statement that the current system may implode on itself, becoming islands of data we are stuck using 20-year-old methods to collect.

\section{The Epocrates Interoperations Research Study}

My proposal to resolve these issues follows, but is prefaced by relevant finding listed in Table 1 from the Epocrates study, which I encourage you to read. ${ }^{6}$

Table 1. Key results from the Epocrates Interoperations Research Study

\begin{tabular}{|c|c|}
\hline Issue & Physicians Responses \\
\hline $\begin{array}{l}\text { Improve } \\
\text { patient care... }\end{array}$ & $\begin{array}{l}\text { - } 8 \text { in } 10 \text { physicians (79\%) agree it is "very important" to } \\
\text { improve the ability of EHRs to access relevant patient } \\
\text { data from other EHRs, and security and privacy of } \\
\text { patient data. } \\
\text { - Ability to engage patients in their treatment } \\
\text { plan/follow-up is the \#3 area of importance for } 77 \% \text { of } \\
\text { physicians who consider this a very important issue. }\end{array}$ \\
\hline $\begin{array}{l}\text { Areas } \\
\text { important to } \\
\text { improve care }\end{array}$ & $\begin{array}{l}\text { - Ability to engage patients in their treatment } \\
\text { plan/follow-up care ( } 96 \% \text { of physicians) } \\
\text { - Ability of EHRs to access relevant patient data from } \\
\text { other EHRs (important to } 96 \% \text { of physicians) }\end{array}$ \\
\hline $\begin{array}{l}\text { Industry } \\
\text { score card }\end{array}$ & $\begin{array}{l}\text { - } 14 \% \text { of physicians give the industry a good grade }(\geq B) \\
\text { on achieving interoperation } \\
\text { - }>6 \text { in } 10 \text { give industry a failing grade ( } D \text { or } F)\end{array}$ \\
\hline
\end{tabular}

However, physicians want to restrict control. Given the potential for universal patient records across disparate care settings, the clear majority of physicians (93\%) believe it is important for them "to have the ability to control visibility and access to particular aspects of a patient record." That statement provides no explanation of this response, However, as any healthcare provider will attest, immediate access to certain information (e.g., a cancer diagnosis) in the absence of perspective might not be in the best interest of a patient.

\section{A Proposed Solution}

The solution proposed addresses standards, storage, accuracy and consistency, access and ownership of this data. There are methods and systems in place that must take a more active and detailed role in this process. Most resistance comes from the vendor's need to modify their structure to become standardized and improve how they use the additional data that becomes available. Some to these proposals relate to security, but 
other concerns focus on vendor cost and the belief that standardizing data will take business advantage away from the vendor.

Standardizing something that will function better for everyone has no impact on independent vendors. Rather, it forces vendors to win or lose based on their product's function, versatility, and ease of use within a new category. That is, the ability to quickly and accurately accumulate and process the most information from the most locations and bring forth the best plan for that patient. The best vendors will get contracts because they help save the most lives.

\section{Standards}

The World Wide Web Consortium is the governing entity that addresses how the Internet functions. There is no excuse for avoiding standardizing data, sharing methods, and agreeing on a uniform set of formats that can be shared by everyone without conflict and endless interfaces.

Currently standardization has had only one leader, the field of imaging. Under DICOM.org there is the DICOM, and PACS standards, which are accepted by every imaging center I know. Now they are enacting a Vendor Neutral Archive-a collective storage of imaging data from all vendors kept under strict standards, allowing access via secure HTML. If the vendor neutral archive was globally adopted, no EMR would have to store a single image. Instead they would store a hyperlink embedded with an encryption key issued to every system that legally has use of the image via a system developed for this purpose.

This decision should be authorized by the owner of the data-the patient. Now the EMR system holds the link and related notes specific for the practice, while the archive holds the image and initial comments from the imaging center. Every EMR gets the same image and comments quickly, in the same quality at an $85 \%$ or better reduction in storage. And when you share data with another center, even with today's interoperability standards, you send the link and not the image as an attachment, reducing the CCDA from megabytes to a few kilobytes while likely providing more and better data. Storage, compute, and transfer resources are reduced $80 \%$ or more-and with it your cost. Expand this to lab data, radiology, pathology, etc., drastically reduced the following components (Table 2 ). 
Table 2. Benefits from reducing the CCDA from megabytes to kilobytes

1. Physically store patient data at your location with reduced costs related to storage, retrieval, and processing

2. Reduce or elimination duplicate data held at different centers with it the risk for inconsistent data or data error

3. Reduction accidental creation of duplicate patient files, and a system built to detect duplicates across the global data set

4. Speed record transfer for referrals or critical care center emergency data requests (trauma centers that depend on the "Golden Hour" *

5. The depth and range of data that can be sent through the existing "XML" formatted CCDA

*Golden Hour: The first hour after occurrence of a traumatic injury, considered the most critical for successful emergency treatment.

\section{Location}

This then adds a second concept, unifying the location of patient data. This carries no greater security risk than the current state and would, in fact, improve security.

Currently, storing this massive amount of data at hundreds of thousands of locations assures two things: inconsistency and overhead. Before going further, consider that you are reading this article on the Internet. You don't know and likely don't care where this article is stored and served. In today's technology, a person in Australia will likely get a different address because copies are spread among secure content management servers worldwide to reduce latency and impact from demand.

Now we have HIEs and HISPs that confirm and relay data. If these locations could collect and store these data under common standards, it would integrate into the service they already provide. For example, they are already designed to securely consolidate massive amounts of data, shorten the path to access, increase the amount of available data, and maintain the certificate system while reducing connections by half. The new cloud model 
would allow research centers to query major data sets to separate short-term servers where they could analyze huge data sets with absolutely no impact on the flow of data to and from each EMR. Global health information then becomes a reality every location.

\section{Ownership}

According to HIPAA, ownership of data pertaining to any patient no matter its location is the patient's, not the hospital, doctor, or government. The inception of the patient portal is the most valuable asset yet devised for EMR. I propose that the portal be a source for the transfer of record data via the CCDA. This is already a capability of the patient portal, but it is not embraced or used efficiently. The portal should have a copy of each patient's fully integrated chart, excluding the last 30 days. This period would allow medical centers to receive lab, imaging, and pathology results, review them and prepare a patient plan of action before the patient sees them and frantically calls the center. These flows directly into my final proposal list below.

\section{Accountable Care Organizations}

The ACO (accountable care organization) concept misses one important component. Half of patient care is in the hands of the patient. It is never just the doctor who is responsible, yet we rate doctors and health institutions on outcomes that are equally or more due to the actions of the patient. Patients must become involved in their health, their care, and the process of recovery from illness. Ratings by ACOs must treat equally the extent of patient participation. This is the only accurate way to fully judge the accountability of success by a medical center. And the numbers collected on participation by the patient should be used by insurance companies to reduce premiums as an incentive, as it also reduces cost over the course of the care plan and beyond.

This is just a start, but without these changes we may never see any of the great promises stated by the ONC (Office of the National Coordinator) and every vendor with an EMR to sell. 


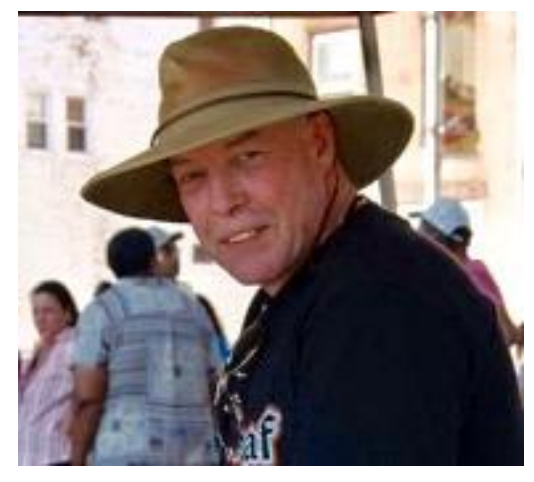

Matthew (Ted) Braid is an IT consultant and network engineer with thirty years' experience in the field of health care technology. He has lived in Baltimore, MD where he has worked at Johns Hopkins and many other progressive medical centers. As someone involved in the evolution of this field from its onset he has developed a passion for promoting the concept that medical technology must become a "Patient First" model for development, and that vendors need to accept the tradeoff of doing what is best for the patient and medicine as a whole, with the need for profit based on being unique and instead simply being better than the competition.

\section{References}

1. "International Classification of Diseases (ICD)". World Health Organization. URL: http://www.who.int/classifications/icd/en/.

2. HealthData Archive. Health Data Volumes Skyrocket, Legacy Data Archives on The Rise. 2017. URL: https://www.healthdataarchiver.com/health-data-volumesskyrocket-legacy-data-archives-rise-hie/.

3. Tuten T. EHR/EMR Facts and Amazing Statistics. Soliant Health. 2017. URL: http://blog.soliant.com/healthcare-it/ehremr-facts-and-amazing-statistics/.

4. Kern C. Healthcare Miscommunication Costs 2,000 Lives And \$1.7 Billion. 2017. Health IT Outcomes. URL: https://www.healthitoutcomes.com/doc/healthcaremiscommunication-costs-lives-and-billion-0001.

5. Swain M, Charles D, Patel V, Search T. Health information exchange among U.S. non-federal acute care hospitals: 2008-1014. National Coordinator for Health Information Technology. 2015. URL: https://www.healthit.gov/sites/default/files/databrief/ONC DataBrief24 HIE Final.pdf.

6. ePocrates. Interoperation Research Study. 2015. URL: 
1806. Braid. A Plea

https://doi.org/10.30953/tmt.v2.22

Page 10 of 10

http://www.epocrates.com/sites/default/files/Interoperation topline 410 15.pdf.

Category: Opinion and Interview

Tags: electronic medical record, EMR, improved patient care, patient, standardization, telehealth 